\title{
LEO PHILIP KADANOFF
}

\section{Maestro who connected physics on multiple scales}

"One might argue, as I do, that the connections among 'laws of nature' at different scales of energy, length or aggregation is the root subject of physics." These words, penned by Leo P. Kadanoff in Nature as part of his obituary for Kenneth G. Wilson, are as apt a description of Kadanoff's scientific legacy as they are of Wilson's. Kadanoff, who passed away on 26 October 2015 after a short illness, often stated that his theory of scaling at phase transitions was his "best work": without doubt, his name will forever be inextricably linked with the idea of block spins. Its inclusion in the title of Wilson's famous 1971 paper is a rare, almost unprecedented circumstance allowed by the editors of Physical Review $B$, who were evidently more aware of the scientific justification than the members of the committee that awarded the 1982 Nobel Prize in Physics solely to Wilson.

Kadanoff was born in 1937 and spent his early life in New York City. He received his PhD from Harvard in 1960 and was a postdoctoral researcher at the Niels Bohr Institute in Copenhagen until he moved to the University of Illinois in 1962. His work there concerned the development of many-body theory techniques, culminating in a short but highly influential monograph with Gordon Baym, which is still widely used today.

Kadanoff found himself, in the mid1960s, working on the problem of phase transitions. It had been known since the work of Lev Landau in 1937 that secondorder phase transitions would exhibit power-law singularities or divergences as the temperature approached the critical point. But 30 years later, the exponents characterizing these singularities, which had been measured or estimated by numerical methods in a number of systems, were in clear disagreement with Landau's predictions.

A crucial contribution was made in 1965 by Benjamin Widom of Cornell University, who showed how the form of the scaling laws near the critical point could be obtained from assuming mathematical homogeneity in the equation of state, but the interpretation of this assumption seemed obscure. Kadanoff made the perspicacious insight that statistical mechanics could be formulated not merely in terms of a partition function relating in

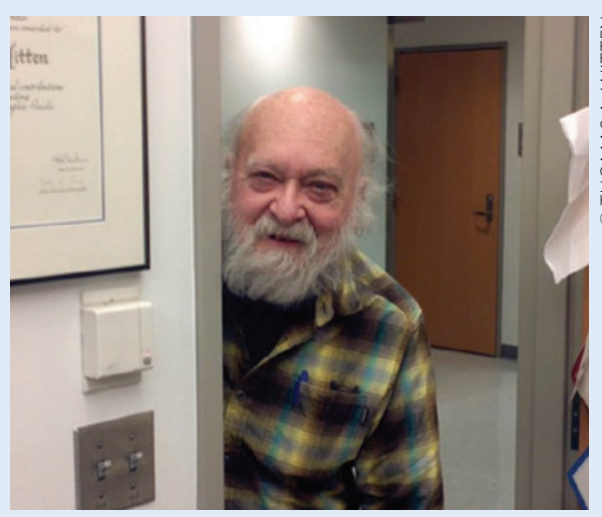

one fell swoop the microscopic Hamiltonian of a system to its system-size thermodynamic free energy, but instead marching one scale at a time from small to large.

In Kadanoff's formulation, degrees of freedom on a small scale would influence degrees of freedom on a larger scale by changing or 'renormalizing' the parameters of the larger-scale Hamiltonian. This process, iterated from atomic level to system-size level, led to a mathematical structure identical to that postulated by Widom. Although Kadanoff's 'block spin construction' related the physics on different scales, it did not on its own provide a calculational method for estimating critical exponents. This last step was undertaken by Wilson through his realization that iteration of a Hamiltonian from one scale to another constituted a form of a dynamical system, whose fixed points and the linearized trajectories around them controlled the phases and phase transitions of the system. The insights derived from Widom, Kadanoff and Wilson's work revolutionized our understanding of the process of constructing theories in statistical mechanics, high-energy physics and beyond.

During the 1980s and 1990s, Kadanoff's research shifted to dynamical system theory. Kadanoff was especially interested in the similarities between diffusion-limited growth processes, such as those responsible for the growth of snowflakes, and the growth of air bubbles in quasi-two-dimensional confined viscous fluids. This class of problems was characterized by exotic scaling behaviour that went well beyond simple fractal ideas, leading to asymptotics beyond all orders and multifractal scalings now part of the standard vocabulary of dynamical systems. Working closely with experimental colleague Albert Libchaber at the University of Chicago, Kadanoff explored thermal convection in an attempt to understand the scaling laws characterizing the most extreme turbulent states. Together they created a vibrant intellectual environment that attracted some of the most brilliant young scientists from around the world, many of whom are now leaders in condensed matter theory and experiment.

In these and other fields, all generally far from equilibrium, the hallmark of Kadanoff's contributions was the deceptive simplicity with which he provided the minimal ingredients that made the problem interesting, but at the same time tractable or approachable. Kadanoff was particularly interested in the way in which simple particle dynamics could, when scaled up to many-particle systems, replicate complex phenomena in the physical world. Thus, he studied how systems of random walkers could simulate air bubbles growing in viscous fluids, and how systems of colliding particles, subject only to conservation laws of mechanics, could be used to simulate complex flows, including turbulence. His fascination with the way in which largescale complex phenomena were the outcome of simple microscopic rules would persist until the end of his career, when he became interested in the historical and philosophical aspects of the applications of renormalization group concepts.

Kadanoff received many honours and was also active in community service, serving as president of the American Physical Society in 2007.

He will be remembered not only for the scientific brilliance that matched the everpresent twinkle in his eyes, but also for the dry humour that peppered his writings and casual conversation. His generosity and hospitality to visitors were legendary, fuelled in part by his creativity in obtaining funding for his enterprises. With his passing, the scientific community has lost one of its most original and influential thinkers, who sought, and frequently found, the simplicity underlying the apparent complexity of the world around us.

\section{NIGEL GOLDENFELD}

Nigel Goldenfeld is in the Department of Physics, University of Illinois at Urbana-Champaign, 1110 W. Green, Urbana, Illinois 61801, USA. e-mail:nigel@uiuc.edu 